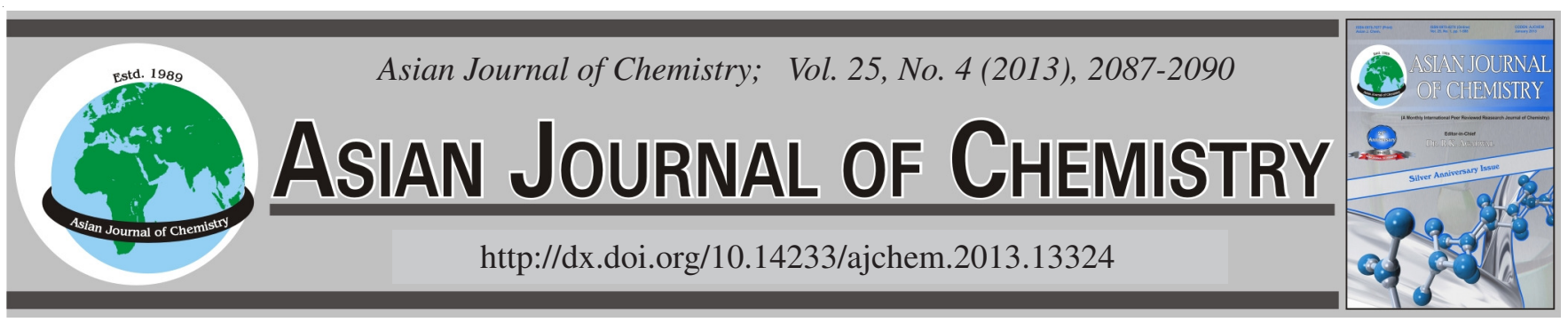

\title{
Green Synthesis of Nanolevel Polyoxometalate of Vanadium with Sulphur as Heteroatom
}

\author{
S. Hari PRASAD ${ }^{1}$, M.K. SReEdhar ${ }^{2}$ and JAdu SAmUel ${ }^{1, *}$
}

${ }^{1}$ Department of Chemistry, Mar Ivanios College, Thiruvananthapuram-695 025, India

${ }^{2}$ Department of Chemistry, University College, Thiruvananthapuram-695 034, India

*Corresponding author: E-mail: prof_jadu@ hotmail.com

(Received: 24 December 2011;

Accepted: 15 October 2012)

AJC-12286

Novel sulphovanadate nanospheres were prepared in a reaction of vanadium(V) oxide and an organic cationic surfactant dodecylpyridinium
chloride (DPC) as structure directing template at the $\mathrm{pH}$ value of 3.0 under ordinary conditions of temperature and pressure by applying
the principles of green chemistry. The specimen was characterized by Fourier transform infrared spectrascopy (FT-IR), which shows that
the nano material consists of S-O, V-O, V=O and S-O-V linkages. Thermogravimetric analysis (TGA) shows that polyoxometalate
contributes $60 \%$ by weight of the sample. The X-ray diffraction analysis (XRD) shows that the particles are amorphous in nature. The
high resolution transmission electron microscopic (HRTEM) analysis shows that the particles are the aggregation of particles of diameter
below $10 \mathrm{~nm}$. The nano scale dimension was further verified by atomic force microscopy.
Key Words: Polyoxometalate, Green synthesis, Surfactant, Nanospheres.

\section{INTRODUCTION}

Polyoxometalates (POM) represent a large class of metal oxygen cluster anions, which exhibit compositional diversity and structural versatility. When the particle size is reduced to nanolevel the properties of the material change to remarkable extent such as conductivity, surface charge and absorption capacity ${ }^{1}$. Nanotechnology now a day attracts great attention due to its tremendous applications in all areas of life $\mathrm{e}^{2}$. Among different areas of inorganic chemistry, metal oxide based polyoxometalate molecular clusters represent a class of inorganic compounds that exhibit unmatched structural versatility ${ }^{3}$. An area of intense research in polyoxometalate chemistry is the synthesis of polyoxovanadates that are known to have attractive chemical and physical properties pertaining to catalysis, biochemistry and material science ${ }^{4}$. Recently the focus in synthesis has been vanadate clusters with reduced or mixed-valence vanadium centers. To this avail one synthetic approach that has been adopted is one of the synergy involving the introduction of organic and anion templating components. The anion is typically imbedded in the vanadate cluster, which can either be covalently or non-covalently bound to the vanadium atoms, used to generate clusters with unique topologies, as in the synthesis of $\mathrm{Na}_{6}\left[\left(\mathrm{PO}_{4}\right) \mathrm{V}(\mathrm{V})_{6} \mathrm{~V}(\mathrm{IV})_{12} \mathrm{O}_{39}\right]_{2} \cdot \mathrm{H}_{3} \mathrm{PO}_{4} \cdot 31 \mathrm{H}_{2} \mathrm{O}$ and $\left[\mathrm{V}(\mathrm{V})_{13} \mathrm{~V}_{3}{ }^{\mathrm{IV}} \mathrm{O}_{42}(\mathrm{CI})\right]^{8-}$, which are examples of covalently bonded and non bonded anions respectively ${ }^{5,6}$. The anionic cluster $\left[\left(\mathrm{PO}_{4}\right) \mathrm{V}(\mathrm{V})_{6} \mathrm{~V}(\mathrm{IV})_{12} \mathrm{O}_{39}\right]^{3-}$ is similar to other molecular polyoxovanadates encapsulating a $\mathrm{VO}_{4}$ template with $6\left\{\mathrm{VO}_{5}\right\}$ square pyramids and 14 octahedral $\left\{\mathrm{VO}_{6}\right\}$ units. However, in contrast to other molecular clusters, the $\left[\left(\mathrm{PO}_{4}\right) \mathrm{V}(\mathrm{V})_{6} \mathrm{~V}(\mathrm{IV})_{12} \mathrm{O}_{39}\right]^{3-}$ clusters are connected by 6 linear V-O-V bridges in to a 3dimensional frame work via the $6\left\{\mathrm{VO}_{5}\right\}$ square pyramids. Nesper and co-workers ${ }^{7}$ reported the sol-gel synthesis of vanadium oxide nanotubes using vanadium alkoxide precursors and primary long chain amines. Polyoxovanadate cluster based solids are attractive inorganic materials due to their structural versatility and potential applications in the areas of catalysis, magnetism, medicine etc. Muller and his groups ${ }^{8}$ assembled several cage like structures $\left[\mathrm{V}_{15} \mathrm{O}_{36}\right]^{5-},\left[\mathrm{V}_{18} \mathrm{O}_{42}\right]^{12-},\left[\mathrm{V}_{19} \mathrm{O}_{45}\right]^{9-}$ and $\left[\mathrm{V}_{34} \mathrm{O}_{82}\right]^{10-}$ in which anions are encapsulated inside the cavity of these spherical clusters. The sizes of the anions $\mathrm{NO}_{3}{ }^{-}$, $\mathrm{Cl}^{-}, \mathrm{Br}^{-}, \mathrm{SCN}^{-}, \mathrm{SO}_{4}{ }^{2-}$ and $\mathrm{PO}_{4}{ }^{3-}$ appear to dictate the structure and geometry of these solids.

Synthesis of polyoxovanadates has classically been done in aqueous solutions or under hydrothermal conditions. Recently new synthetic routes are being explored for the formation of vanadate clusters from organic media with organically soluble vanadium precursors. One example of a vanadium cluster synthesized from organic solvent which has been reported by Hong et al. ${ }^{9}$, is $\left\{\mathrm{V}(\mathrm{IV})_{2} \mathrm{~V}(\mathrm{~V}){ }_{12} \mathrm{O}_{36} \mathrm{Cl}\right\}^{5-}$ as a tetraethylamine salt that has blue luminescence. In this reaction $\mathrm{VO}_{2 \text { (acac) }}$ was used as a source of soluble $\mathrm{VO}_{2}{ }^{+}$in acetonitrile. The cluster was then crystallized from a DMF solution with $\left[\mathrm{Et}_{4} \mathrm{~N}\right]_{5}\left[\mathrm{~V}(\mathrm{IV})_{2} \mathrm{~V}(\mathrm{~V})_{12} \mathrm{O}_{36} \mathrm{Cl}\right]$ being air stable in both solution 
and in the solid state. The cluster is built up from $14\left\{\mathrm{VO}_{5}\right\}$ square pyramids however, unlike other polyoxovanadate clusters, it is an open cage with two holes in the surface reminiscent of a basket. The non bonded chloride ion occupies the centre of the cavity, with an average vanadium-chloride distance of $3.496 \AA$ and may offer a path for exchange of the chloride anion with other anions.

In a broad sense green synthesis includes any chemical process or technology that improves the environment and thus our quality of life ${ }^{10}$. Our attempt was to synthesize the nano dimensional polyoxovanadate containing sulphur hetero atom from aqueous solutions. Here we present the formation of nano sized inorganic polyoxovanadate clusters with spherical shape by applying the principles of green chemistry.

\section{EXPERIMENTAL}

Synthesis of nanosulphovanadate by incorporating sulphate units to vanadium frame work of sodium vanadate using dodecylpyridinium chloride (DPC) as templating agent is investigated. All the chemicals used are of analytical reagent grade and used as received without any further purification. Doubly distilled water was used throughout the experiment for the synthesis of sulphovanadate nano particles.

Preparation of sulphovanadate nanospheres: Vanadium(V) oxide was used as vanadium source. Dodecylpyridinium chloride was used as the structure directing template for synthesis ${ }^{11,12}$. Weighed amount of vanadium(V) oxide was dissolved in an aqueous solution of sodium hydroxide by stirring and warming (molar ratio of $\mathrm{NaOH} / \mathrm{V}_{2} \mathrm{O}_{5}$ $=2$ ) thoroughly and it was treated as solution $\mathrm{X}$, weighed amount at dodecylpyridinium chloride was dissolved in 1.0 $\mathrm{M}$ solution of sulphuric acid (mass ratio of $\mathrm{DPC} / \mathrm{V}_{2} \mathrm{O}_{5}=0.25$ ) and it was treated as solution Y. Solution Y was added to solution $\mathrm{X}$ with stirring. The resulting $\mathrm{pH}$ of the solution was found to be 3 . Then the mixture is stirred continuously for $24 \mathrm{~h}$. The mixture is then kept undisturbed for $48 \mathrm{~h}$. It is then filtered, washed, dried and weighted. The sample was found to be orange brown in colour.

Detection method: With the help of modern sophisticated and high resolution instrumental techniques, the fundamental chemistry and structure of polyoxometalate were studied. Particle size analysis was done on a Malvern-Zetasizer Nano ZS particle size analyzer. Submicron particle sizes are measured by observing the scattered light from these particles, determining the diffusion speed and deriving the size from this using Stokes-Einstein relationship by dynamic light scattering method. Initial studies were conducted with Scanning electron microscope to measure the size and morphology of the product. FTIR study of the sulphovanadate sample was recorded on $\mathrm{KBr}$ pellets (Shimadzu spectrophotometer). Thermal analysis was performed on Universal V3.9A TA from 293-1263 K at a heating rate of $10{ }^{\circ} \mathrm{C} / \mathrm{min}$ in nitrogen atmosphere. X-Ray diffraction (XRD) data were recorded on X'Pert Software diffractometer by using $\mathrm{CuK}_{\alpha}$ radiation $(\mathrm{x}=1.5418 \AA$ ). High resolution transmission electron microscopic (HRTEM) studies were done on Philips CM 20 electron microscope operated at $100 \mathrm{kV}$. Before HRTEM measurements the specimen were ground, sonicated in absolute alcohol for $45 \mathrm{~min}$ and deposited on carbon-coated copper grids. The analysis was done at different scales as 20, 50, 100 and $200 \mathrm{~nm}$. The atomic force microscopic (AFM) study was done by dispersing the sample in absolute alcohol and deposited on a silicon substrate using the principle of cantilever.

\section{RESULTS AND DISCUSSION}

Successful covalent linking of S-O-V could be proven by infrared experiments. The corresponding FT-IR spectra are presented in Fig. 1. The peaks at $1171,1125 \mathrm{~cm}^{-1}$ are assigned to asymmetric stretching of $\mathrm{SO}_{4}{ }^{2-}$ groups. The peaks at 1022 $\mathrm{cm}^{-1}$ represent the symmetric stretching of $\mathrm{SO}_{4}{ }^{2-}$ group ${ }^{13}$. The peaks at $579-529 \mathrm{~cm}^{-1}$ are assigned to the symmetric bending of $\mathrm{SO}_{4}{ }^{2-}$ group. The peak $833 \mathrm{~cm}^{-1}$ is due to $\mathrm{S}-\mathrm{O}-\mathrm{V}$ bending. The peak around $959 \mathrm{~cm}^{-1}$ is due to $\mathrm{V}=\mathrm{O}$ stretch and a weak peak around $683 \mathrm{~cm}^{-1}$ is due to V-S-O. Two absorption bands of 2922 and $2853 \mathrm{~cm}^{-1}$ correspond to aliphatic $\mathrm{C}-\mathrm{H}$ vibrations of dodecylpyridinium chloride. The broad peak around 3439 $\mathrm{cm}^{-1}$ is due to $\mathrm{O}-\mathrm{H}$ stretching vibrations of adsorbed water on the surface of the powdered sample.

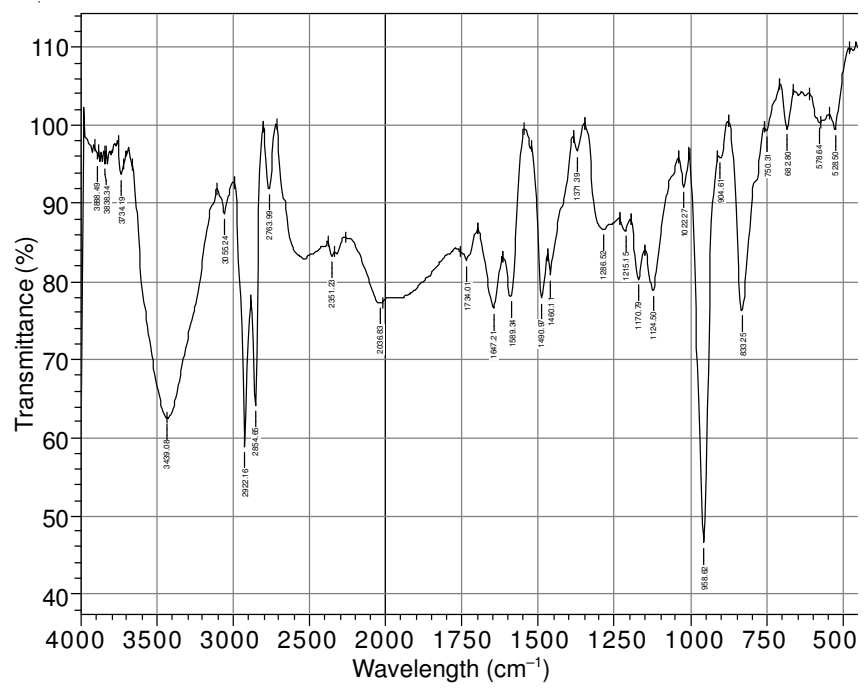

Fig. 1. FTIR Spectrum of the sample of sulphovanadate nanospheres recorded on $\mathrm{KBr}$ pellets

The thermal behaviour of the sample was investigated by TGA as shown in Fig. 2. The small weight loss $(<5 \%)$ up to $523 \mathrm{~K}$ due to the removal at surface adsorbed water molecules. The weight loss from 523-823 $\mathrm{K}$ is due to the loss of DPC incorporated with the nanospheres. The weight loss due to the removal of DPC is $40 \%$. The material is thermally stable up to a temperature of about $340-350 \mathrm{~K}$ and can be used for various processes up to that temperature.

The X-ray diffraction analysis shows that the particles are below crystalline dimension and hence amorphous in nature. The morphology of the nano particles were investigated by HRTEM analysis. (Fig. 3a-b) shows the particles are spherical in shape with diameter $10-100 \mathrm{~nm}$ and they are made up of multiply twinned particles (MTPs).

The morphology of the nano particles were further verified by atomic force microscopic (AFM) analysis. Fig. 4a shows the presence of nanospheres of diameter $25.6 \mathrm{~nm}$ while Fig. 4b shows the particles at diameter $19 \mathrm{~nm}$ and Fig. 4c shows 


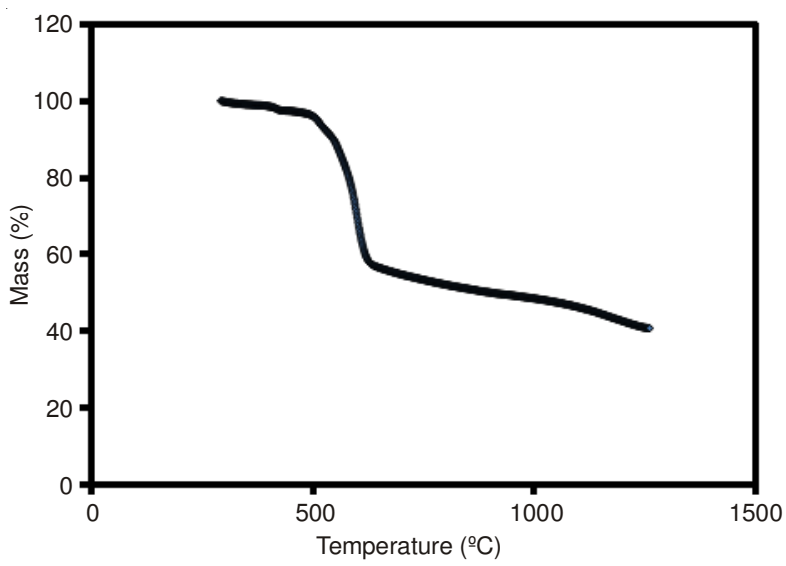

Fig. 2. TG Analysis of nanospheres of sulphovanadate at a heating rate of 100 c per min in $\mathrm{N}_{2}$ atmosphere
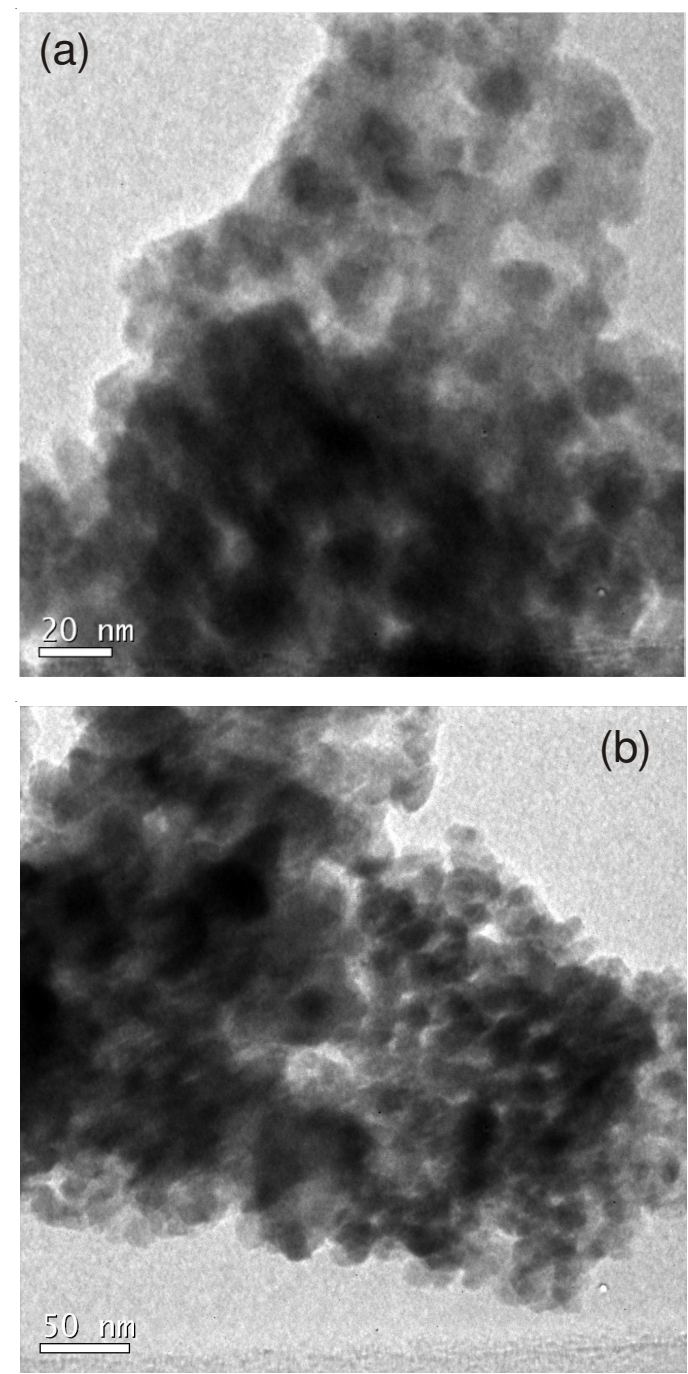

Fig. 3. (a) HRTEM Analysis of nanosulphovanadate by sonicating in absolute alcohol for 45 min using Philips CM 20 electron microscope particles of diameter $20 \mathrm{~nm}$. (b) particles of diameter $50 \mathrm{~nm}$

that the particles are of diameter $50 \mathrm{~nm}$. The nanospheres of sulphovanadate above $10 \mathrm{~nm}$ are formed by the growth of nano particles of SVO having diameter 1-6 nm. The particles sizes at different time intervals of stirring shows that during $24 \mathrm{~h}$ stirring the growth of particles occurs to $10-100 \mathrm{~nm}$ by the
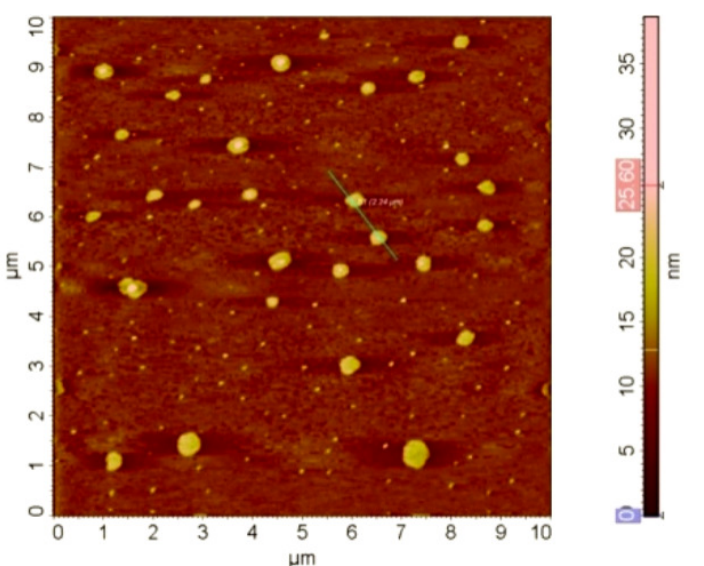

(a)
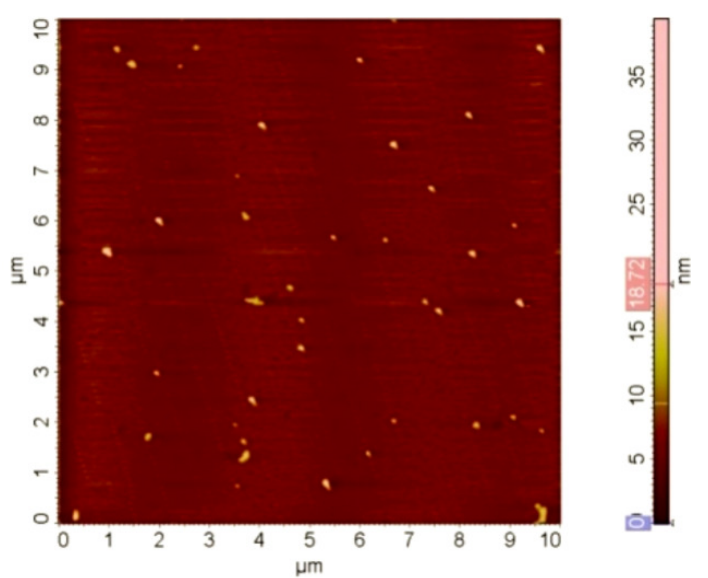

(b)
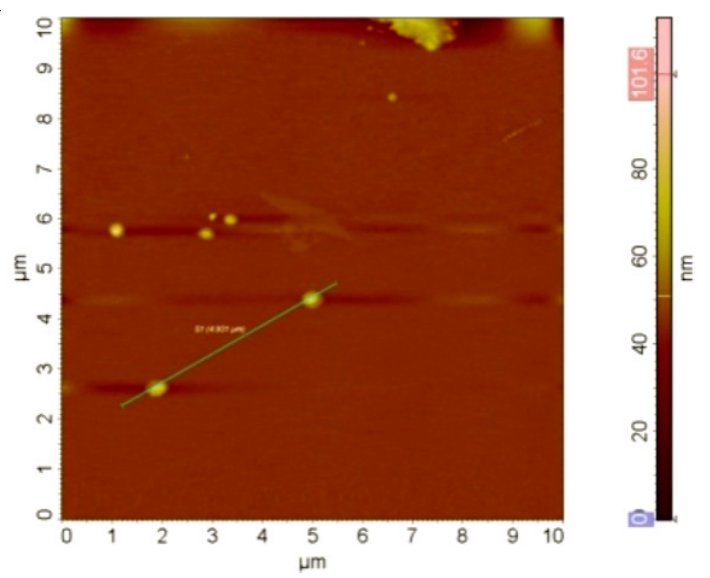

(c)

Fig. 4. (a) AFM of the sample of sulphovanadate having diameter 25.6 $\mathrm{nm}$, obtained by dispersing in absolute alcohol and then deposited on a silicon substrate, (b) $19 \mathrm{~nm}$ and (c) $50 \mathrm{~nm}$

growth in the diameter of nanospheres due to the accumulation at multilayers of dodecylpyridinium chloride at the surface around the growing spheres and blocking access of particles core. With high concentration of dodecylpyridinium chloride the particles is like micelles.

Effect of temperature in the formation of nanosulphovanadate: Chemical reactions are influenced by different variables like temperature, pressure, concentration and catalysts. In the formation of nanosulphovanadate we focus the effect 
of temperature. This can be obtained by combining the law of mass action and Nernst equation from electrochemistry

$$
\text { i.e. } \quad \operatorname{In} \mathrm{K}=\frac{\Delta \mathrm{S}^{\mathrm{o}}}{\mathrm{R}}-\frac{\Delta \mathrm{H}^{\mathrm{o}}}{\mathrm{R}}\left(\frac{\mathrm{I}}{\mathrm{T}}\right)
$$

For an endothermic reaction $\Delta \mathrm{H}^{\circ}$ is positive, an increase in temperature will increase the value of $\mathrm{K}$. But for an exothermic reaction the value of $\mathrm{K}$ can be increased by lowering the temperature.

The effect of temperature in the formation of nanosulphovanadate is as shown in the Fig. 5, which indicates the yield increases as the temperature increases up to $303 \mathrm{~K}$ and then remains more or less constant. But after $308 \mathrm{~K}$ the coagulation increases and particles become bigger in size. According to the thermodynamic principles a process will be spontaneous, if $\Delta \mathrm{G}$ is negative and $\Delta \mathrm{S}$ is positive at all values of temperature. When particle sizes are reduced to nanolevel the entropy also increase $(\Delta \mathrm{S}=+\mathrm{ve})$ i.e., process is spontaneous at all conditions of temperature. In the present work, in order to maintain the particle size to nano level, the reactions were carried out at ordinary temperature obeying the principles of green chemistry $^{14,15}$

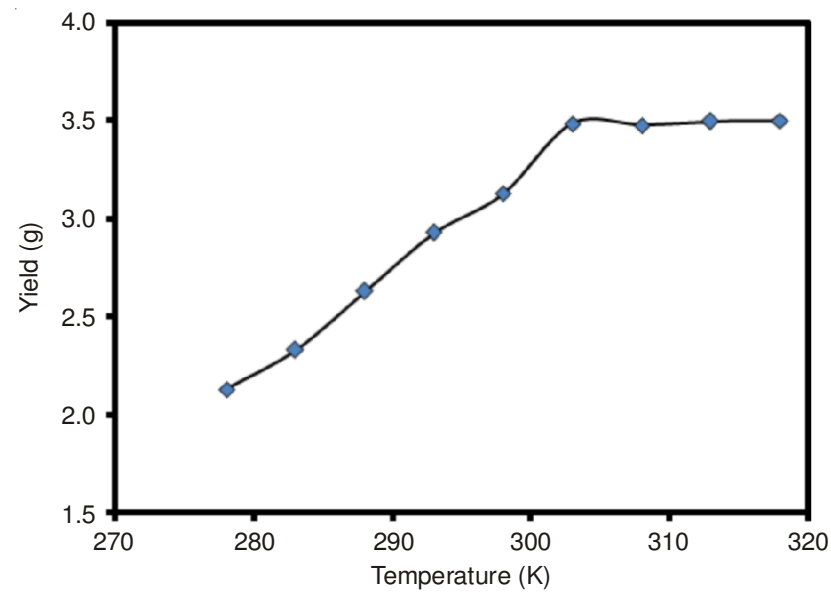

Fig. 5. Effect of temperature in the yield of nanosulphovanadate

Effect of surfactant concentration: In the formation of sulphovanadate the concentration of the surfactant dodecylpyridinium chloride has a dual role to control the particle size to nano level and to produce maximum yield. In the present work, the maximum yield of nanosulphovanadate is obtained by maintaining the mass ratio of DPC/ $\mathrm{V}_{2} \mathrm{O}_{5}=0.25$. The surfactant molecules prevent agglomeration of reactant molecules and maintain them in nano scale. For low mass ratio (DPC/ $\mathrm{V}_{2} \mathrm{O}_{5}=0.05$ ), no precipitate was obtained. But by increasing in the $\mathrm{DPC} / \mathrm{V}_{2} \mathrm{O}_{5}$ ratio to 0.5 , results in the formation of sulphovanadate spheres with larger diameters. The protonated surfactant moieties influence the morphology of nano polyoxometalate structures.
Solubility of the material: The material is insoluble in water, alcohol, ether and acetone at ordinary temperature. It is one of the remarkable property of nanosulphovanadate compared to the ordinary sulphovanadate and can be used for various reactions or process like adsorption in organic or inorganic media ${ }^{16}$.

\section{Conclusion}

A highly efficient method for the synthesis of nano-sized sulphovanadate with remarkable yield by applying the principles of green chemistry is developed. The material is of great importance due to its insolubility in water, alcohol, ether and acetone and can be used for environmentally beneficial process like adsorptions or reactions like catalysis in these media. The results obtained also represent a significant step for the better understanding of growth mechanism of sulphovanadate nanospheres and may lead to better synthesis, properties and applications polyoxometalates of other addenda atoms.

\section{ACKNOWLEDGEMENTS}

The authors are thankful to University Grant Commission, New Delhi, India for the financial support and NIIST, Thiruvananthapuram, Kerala, India for lending the analytical facilities.

\section{REFERENCES}

1. M. Jiang, Enbowang, Z. Kang, S. Lian, A. Wu and Z. Li, J. Mater. Chem., 13, 647 (2003).

2. G. Maayan, R. Popovitz-Biro and R. Neumann, J. Am. Chem. Soc., 128, 4968 (2006).

3. A. Nisar, J. Zhuang and Xunway, Chem. Mater., 21, 3745 (2009).

4. De-L. Long, E. Burkholder and L. Cronin, Chem. Soc. Rev., 36, 105 (2007).

5. W.B. Yang, C.H. Lu, Q.Z. Zhang, S.M. Chen, X.P. Zhan and J.H. Liu, Inorg. Chem., 42, 7309 (2003).

6. M.I. Khan, S. Ayesh, R.J. Doedens, M.H. Yu and C.J. O'Connor, Chem. Commun., 4658 (2005).

7. H.J. Muhr, F. Krumeich, U.P. Schonholzer, F. Bieri, M. Neiderberger, L.J. Gauckler and R. Nesper, Adv. Mater., 12, 231 (2000).

8. J. Thomas, S. Sharma, S.E. Lofland, K.V. Ramanujachary and A. Ramanan, J. Chem. Sci., 118, 79 (2006).

9. L. Chen, F.L. Jiang, Z.Z. Lin, Y.F. Zhou, C.Y. Yue and M.C. Hong, J. Am. Chem. Soc., 127, 8588 (2005)

10. R. Sanghi and M.M. Srivastava, Green Chemistry Environment Friendly Alternatives,Narosa Publishing House, Daryaganj, New Delhi, end. 2, p. 5 (2007)

11. M. Asnani, J. Thomas, P. Sen and A. Ramanan, Mater. Res. Bull., 42, 686 (2007).

12. J. Thomas, K.R. Kannan and A. Ramanan, J. Chem. Sci., 120, 529 (2008).

13. K. Nakamoto, Infrared and Raman Spectra of Inorganic and Cordination Compounds, John Wiley \& Sons, Inc., New York, edn. 4, p. 325 (1986).

14. X. Chen, S. Lin, L. Chen, X. Chen, C. Liu, J. Chen and L. Yang, Inorg. Chem. Commun., 10, 1285 (2007).

15. Q. Deng, W. Zhou, X. Li, Z. Peng, S. Jiang, M. Yue and T. Cai, J. Mol. Catal. A 262, 149 (2007).

16. L.W. Ting, H. Chengzhi and Malu, Sci. China Ser. B-Chem., 52, 2156 (2009). 